\title{
Validity and reliability of a linear positional transducer across commonly practised resistance training exercises
}

Harry F. Dorrell, Joseph M. Moore, Mark F. Smith \& Thomas I. Gee

To cite this article: Harry F. Dorrell, Joseph M. Moore, Mark F. Smith \& Thomas I. Gee (2018):

Validity and reliability of a linear positional transducer across commonly practised resistance training exercises, Journal of Sports Sciences, DOI: 10.1080/02640414.2018.1482588

To link to this article: https://doi.org/10.1080/02640414.2018.1482588

Published online: 31 May 2018.

Submit your article to this journal $\sqsubset$

Щ Article views: 79

Q View related articles ๘

View Crossmark data ¿ 


\title{
Validity and reliability of a linear positional transducer across commonly practised resistance training exercises
}

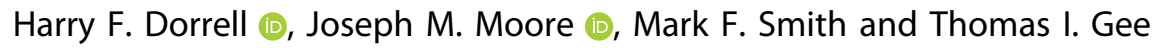 \\ Human Performance Centre, School of Sport and Exercise Science, College of Social Sciences, University of Lincoln, Lincoln, UK
}

\section{ABSTRACT}

This study investigated the validity and reliability of the GymAware PowerTool (GPT). Thirteen resistance trained participants completed three visits, consisting of three repetitions of free-weight back squat, bench press, deadlift ( $80 \%$ one repetition maximum), and countermovement jump. Bar displacement, peak and mean velocity, peak and mean force, and jump height were calculated using the GPT, a threedimensional motion capture system (Motion Analysis Corporation; $150 \mathrm{~Hz}$ ), and a force plate (Kistler; $1500 \mathrm{~Hz}$ ). Least products regression were used to compare agreeability between devices. A within-trial one-way ANOVA, typical error (TE; \%), and smallest worthwhile change (SWC) were used to assess reliability. Regression analysis resulted in $R^{2}$ values of $>0.85$ for all variables excluding deadlift mean velocity $\left(R^{2}=0.54-0.69\right)$. Significant differences were observed between visits $3-2$ for bench press bar displacement $(0.395 \pm 0.055 \mathrm{~m} ; 0.383 \pm 0.053 \mathrm{~m})$, and deadlift bar displacement $(0.557 \pm 0.034 \mathrm{~m}$; $0.568 \pm 0.034 \mathrm{~m})$. No other significant differences were found. Low to moderate TE $(0.6-8.8 \%)$ were found for all variables, with SWC ranging $1.7-7.4 \%$. The data provides evidence that the GPT can be used to measure kinetic and kinematic outputs, however, care should be taken when monitoring deadlift performance.
ARTICLE HISTORY

Accepted 24 May 2018

KEYWORDS

Velocity based training; GymAware; reliability;

strength and conditioning; power

\section{Introduction}

Resistance training is an essential stimulus for the development of muscular strength and power, and is considered fundamental to many athlete's periodised regimes (Harries, Lubans, \& Callister, 2012 , 2015). One approach to ensure the effectiveness of such regimes is through the monitoring of acute training variables. Traditionally, variables were limited to the volume and intensity of a given movement, however, as technology and training practices have developed, access to more complex variables such as lift velocity and force have become available (Peterson, Rhea, \& Alvar, 2004). These variables have been shown to offer insight into an athlete's development and have a wide range of uses in the design and monitoring of periodised regimes (Pereira \& Gomes, 2003).

The velocity at which a given lift is performed is directly related to both the force-velocity relationship of the athlete, and their current state of fatigue (Haff, 2012; Sanchez-Medina \& González-Badillo, 2011). Therefore, the measurement of lift velocity allows insight into the athlete's current physiological status (Conceição, Fernandes, Lewis, Gonzaléz-Badillo, \& Jimenéz-Reyes, 2016; González-Badillo et al., 2015; GonzálezBadillo \& Sánchez-Medina, 2010). This allows informed decisions to be made on factors such as proposed training volume, prescribed training loads, and regime progression (Kraemer \& Ratamess, 2004). This is often done through use of athlete velocity profiling, facilitating the prediction of one repetition maximum, and the prescription of individualised velocity training zones (González-Badillo \& Sánchez-Medina, 2010;
Jidovtseff, Harris, Crielaard, \& Cronin, 2011; Kraemer \& Ratamess, 2004; Sánchez-Medina, Pallarés, Pérez, MoránNavarro, \& González-Badillo, 2017). Due to the potential implication of these measures, the accurate and reliable assessment of these performance variables is essential.

Traditionally, the direct acquisition of these performance variables required the use of a force plate and/or high speed video analysis, limiting research to specialised facilities due to labour- and resource-intensive protocols (Lamas et al., 2012). Whilst these methods are widely considered "gold standard" in terms of performance assessments, the transferability to an applied setting has been questioned (Cronin, Hing, \& Mcnair, 2004). To overcome this, kinematic systems, including linear positional transducers (LPTs), have become increasingly popular tools for quantifying the force, power and velocity outputs of resistance training exercises (Argus, Gill, \& Keogh, 2012; González-Badillo et al., 2015; Sánchez-Medina et al., 2017).

Commercially available LPTs use a tether attached directly to the athlete or weightlifting bar, enabling real-time collection of time-displacement data. This data, along with inputted mass (athlete and/or system), is then used to derive further variables such as, velocity, acceleration, force, and power. Research has utilised LPTs across a range of resistance training movements, with the aim of measuring and applying performance variables, in both training and testing environments (Argus, Gill, Keogh, Hopkins, \& Beaven, 2010; Conceição et al., 2016; Cormie, Mccaulley, Triplett, \& Mcbride, 2007; 
Drinkwater, Moore, \& Bird, 2012). Whilst these monitoring tools are becoming prevalent within both applied and research environments, the validity and reliability of specific devices has yet to be fully examined within the literature.

The GymAware PowerTool (GPT) is a commercially available LPT, which has been used within an array of research studies evaluating velocity (Argus, Gill, Keogh, \& Hopkins, 2011), acceleration (Beaven, Cook, Kilduff, Drawer, \& Gill, 2012), force (Crewther, Kilduff, Cunningham, Cook, \& Yang, 2010), and power (Argus et al., 2012), across a range of resistance training movements. However, minimal research exists exploring the validity and intra-visit reliability of the device. Drinkwater, Galna, McKenna, Hunt, and Pyne (2007) evaluated the validity of the GPT, during free-weight bench press, and Smithmachine back squat and bench throw. Eccentric and concentric peak and mean power output were calculated through use of the GPT and validated against two-dimensional video data. Validity was quantified through use of standard error of measurement, and coefficient of variation (3.6-14.4 W, 1.0$3.0 \%$, respectively). The relationships between the criterion device (video) and the GPT were evaluated using Pearson's product moment, producing strong correlations $(r \geq 0.97)$. Whilst the data presented suggests high levels of validity, the use of manually calibrated, two-dimensional video capture limits the practical applications of this research due to an increased risk of both systematic and random error.

Crewther et al. (2011) investigated the validity of the GPT during weighted squat jumps $(20 \mathrm{~kg}, 40 \mathrm{~kg}, 60 \mathrm{~kg}$, and $80 \mathrm{~kg}$ ). Concentric peak force and power of twelve trained participants were assessed via comparisons of the GPT and a force platform (Kistler, Switzerland). Relative validity was quantified using least squares regression ( $r=0.59-0.87)$, with BlandAltman plots revealing high random error across all assessed resistances for peak force $(20 \mathrm{~kg}: \pm 579 \mathrm{~N} ; 40 \mathrm{~kg}: \pm 255 \mathrm{~N}$; $60 \mathrm{~kg}: \pm 255 \mathrm{~N} ; 80 \mathrm{~kg}: \pm 414 \mathrm{~N}$ ), and peak power $(20 \mathrm{~kg}$ : $\pm 879 \mathrm{~W} ; 40 \mathrm{~kg}: \pm 611 \mathrm{~W} ; 60 \mathrm{~kg}: \pm 748 \mathrm{~W} ; 80 \mathrm{~kg}: \pm 762 \mathrm{~W})$. The authors suggested that the error was likely due to the differing methods between measuring devices. Force plates measure the centre of mass directly, while the GPT differentiates this variable from collected time-displacement data. Thus, any body movement occurring independently of the bar, potentially affecting centre of mass, will be missed by the GPT (Crewther et al., 2011).

The limitations of the above research highlight a clear need for further investigation into the validity of the GPT. Furthermore, to our knowledge, the intra-visit reliability of the GPT, set within a resistance-based exercise paradigm, has yet to be examined. Producing outcome measures that have addressed these issues would allow researchers and practitioners to make informed decisions about use of the GPT within athletic programme design and monitoring. Researchers should endeavour to ensure data collected is applicable to common strength and conditioning practice. This should be achieved by assessing the validity and reliability of the GPT on commonly employed lifts, which have a range of techniques, lift distances, and velocities. Therefore, the aims of this research were to investigate and establish the validity of the GPT against integrated criterion devices, and to assess the reliability of the GPT over three repeated visits.
These were addressed by evaluating displacement, velocity, and force outputs of the back squat, bench press, deadlift (all free-weight), and countermovement jump (CMJ), within a trained population group.

\section{Methods}

\section{Participants}

Thirteen resistance trained participants (mean $\pm \mathrm{SD}$, age: $26.5 \pm 4.8$ years, height: $1.74 \pm 0.10 \mathrm{~m}$, body mass: $81.9 \pm 12.1 \mathrm{~kg}$ ), volunteered and consented to take part in the study. All participants were free from injury and had at least two years of resistance training experience prior to the start date. All participants had experience of the movements required prior to acceptance on the study. Protocols were submitted to, and approved by, the local ethical review board at the institution, in line with the Helsinki Declarations for research with human volunteers.

\section{Procedures}

On their initial visit, participants completed a one repetition maximum test protocol (1RM) for back squat, bench press, and deadlift in accordance with guidelines established by the National Strength and Conditioning Association (Baechle \& Earle, 2000). This was used as both a familiarisation session, and to obtain 1RM data. Following this, participants completed three further visits, interspaced with a minimum of 96 hours rest (maximum 120 hours). Upon each visit, a standardised warm-up was overseen, consisting of five minutes of stationary cycling (60 rpm, $60 \mathrm{~W})$, followed by mobility and practice movements with the powerlifting bar (Eleiko, Sweden; $20 \mathrm{~kg}$ ). Participants subsequently completed the testing protocol consisting of three repetitions of free-weight back squat, bench press, and deadlift (completed at $80 \% 1 \mathrm{RM}$ ), followed by three CMJs. Trial order was consistent for all visits. For all lifts, participants were instructed to maintained eccentric control, before generating maximal force during the concentric phase of each repetition. For the CMJ, participants were instructed to keep their hands in contact with their hips (iliac crest) throughout the movement.

For each trial completed, the GPT was attached to the powerlifting bar (back squat, bench press, and deadlift) or athlete (CMJ), with the participant standing on a force plate (Kistler, Switzerland). For the back squat and bench press, the GPT tether attachment site (GA) was located $10 \mathrm{~cm}$ from the end of the bar, with this being centred during the deadlift (Figure 1). For the CMJ, the GPT tether was attached directly to the athlete's midriff (in line with the naval) via a Velcro fastening. Time-displacement data were measured and recorded by the GPT. Bar kinematics were recorded using a five-camera three-dimensional motion capture system (Rapture-E, Motion Analysis Corporation, Santa Rosa, CA; $150 \mathrm{~Hz}$ ). Three passive retro-reflective markers $(12 \mathrm{~mm}$ diameter) were used, two placed on the powerlifting bar (diametric ends; Figure 1), and one on the GA. Force data were collected $(1500 \mathrm{~Hz})$ during all movement trials except bench press. 

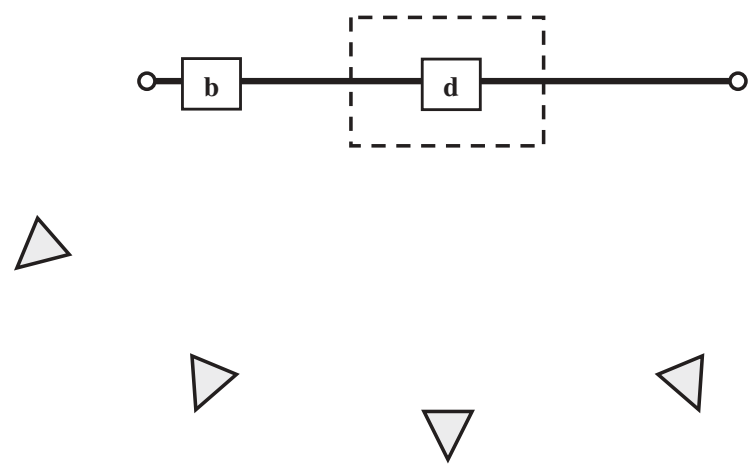

Figure 1. Data capture set-up, detailing force plate (- - ), powerlifting bar (-), cameras $(\nabla)$, markers (o), and GymAware attachment site (back squat and bench press: b; deadlift: d).

\section{Data processing}

Marker positions were identified using Cortex (v5.3.1, Motion Analysis Corporation, Santa Rosa, CA) and analysed using custom written MATLAB code (R2016a, MathWorks, Natick, MA). Marker data were smoothed using a zero lag $2^{\text {nd }}$ order Butterworth low-pass filter, with a cut-off frequency of $6 \mathrm{~Hz}$. For each trial a virtual midpoint (VM) was created by taking the mean position of the diametric markers, representing the true centre of the bar. The VM and GA position data from the motion capture system were used to represent bar, and GymAware tether movement, respectively. Simultaneous collection of data were completed by the GPT, and analysed via the built-in GymAware Pro (GAP) software (Kinetic Performance Technology, Canberra, Australia). The GAP software automatically down-samples collected data to 50 points a second, removing the need for smoothing.

For all movements, the concentric phase of each trial was analysed from the onset of movement to a predefined end point. The GAP software automatically detects the start of the concentric phase as the first moment the tether data increases $(\geq 0.30 \mathrm{~mm}$ ) above its lowest vertical position. End points are defined as the point of greatest vertical displacement occurring after the predefined start. To minimise differences due to identification of start and end points, analyses closely matching the GAP software were used within the marker positional analysis. For the back squat and bench press, the start of the concentric phase of the movement was identified as the frame at which the vertical position was at its lowest point. For deadlift, the start of the concentric phase was identified as the first frame the vertical position was greater than $0.30 \mathrm{~mm}$ above the starting position. For all movements, the end point was identified as the point where marker vertical position was at its highest following the identified start point.

Barbell displacement was measured as the vertical distance between the predefined start and end of each trial. The first and second derivatives of displacement data were calculated to provide bar velocity and acceleration, respectively. The differentiation method used by the GAP software takes the difference between two adjacent points, divided by the change in time. Subsequently, force is calculated by multiplying acceleration by inputted mass. In contrast, the central difference method was used for the differentiation of marker data.
This method provides an estimate of the slope of the tangent at a single point using the preceding and succeeding data (Hamill, Knutzen, \& Derrick, 2015). This allows the calculation of instantaneous velocity and acceleration at a specific time point, rather than the average between two points. Comparative force data was obtained via direct measurement from the force plate. Peak and mean values were extracted for velocity and force enabling comparison between collection methods.

To calculate jump height, the GAP software took the difference between vertical displacement of the tether from a predefined start point (participant standing on toes), to the point of the highest vertical position. For comparison, jump height was calculated according to the impulse-momentum relationship, using change in centre of mass velocity from the captured force plated data.

\section{Statistical analysis}

For all variables, the within-trial (visit) data for each participant were averaged and the mean result used for statistical analysis. Statistical analyses were conducted using SPSS 22.0 (Chicago, IL) with the alpha level for significance set at $a=0.05$.

Validity between the criterion (motion capture and force plate data) and GPT calculated variables were evaluated using least products regression and expressed as an $\mathrm{R}^{2}$ value. To assess reliability, a within-trial one-way ANOVA test with repeated measures $(1 \times 3)$ were conducted to examine the between visit differences across all GPT variables. Within-participant variation was reported as typical error (TE) and displayed as a percentage (90\% confidence interval) following completion of a consecutive pairwise analysis spreadsheet (Hopkins, 2015). Smallest worthwhile change (SWC) was calculated by multiplying the mean between-participant standard deviation by 0.2 (representing a small Cohen's effect size; Drinkwater et al., 2005) and presented as both absolute and relative values. Estimated sample-size requirements for subsequent research were calculated using the reported TE and SWC, using methods described by Hopkins (2000). As the GPT derives all performance variables from timedisplacement data, the power-analyses were only run on this variable across movements.

\section{Results}

\section{Validity}

Correlations between the GPT and the GA and VM sites for all kinematic variables resulted in an $R^{2} \geq 0.99$ for back squat and $R^{2} \geq 0.91$ for bench press (excluding VM displacement; $R^{2}=0.85$ ). For deadlift, correlations resulted in an $R^{2} \geq 0.92$ for all kinematic variables, barring mean velocity for both GA and VM sites; $R^{2}=0.54$ and $R^{2}=0.69$, respectively (Table 1 ; Figure 2 ).

Correlations for back squat kinetics resulted in an $R^{2} \geq 0.99$ for peak and mean force (mean difference \pm SD: peak force $=136.4 \pm 86.0 \mathrm{~N}$; mean force $=28.0 \pm 39.1 \mathrm{~N}$ ). Similarly, strong correlations of $R^{2}=0.97$ and $R^{2}=0.94$ for peak and mean force respectively (peak force $=-14.5 \pm 69.0 \mathrm{~N}$; mean force $=52.0 \pm 74.6 \mathrm{~N}$ ) were found for deadlifts. For CMJ, correlations between the GPT and calculated jump height had $\mathrm{R}^{2}=0.88$. 
Table 1. Mean difference of kinematic variables between the GymAware power tool (GPT) and the GymAware attachment site (GA) and virtual midpoint (VM) sites [means $\pm S D\left(R^{2}\right)$ ]

\begin{tabular}{lrr}
\hline & Displacement $(\mathrm{m})$ & Peak velocity $\left(\mathrm{ms}^{-1}\right)$ \\
\hline Back squat & & \\
GA & $-0.009 \pm 0.005(0.99)$ & $0.005 \pm 0.007(0.99)$ \\
VM & $-0.019 \pm 0.010(0.99)$ & $-0.022 \pm 0.025(0.99)$ \\
Bench press & & \\
GA & $-0.009 \pm 0.009(0.98)$ & $0.029 \pm 0.010(0.99)$ \\
VM & $0.001 \pm 0.022(0.85)$ & $0.009 \pm 0.007(0.99)$ \\
Deadlift & & $0.026(0.91)$ \\
GA & $-0.016 \pm 0.009(0.94)$ & $0.013(0.99)$ \\
VM & $0.001 \pm 0.010(0.92)$ & $0.004 \pm 0.004(0.99)$ \\
\hline
\end{tabular}

a.

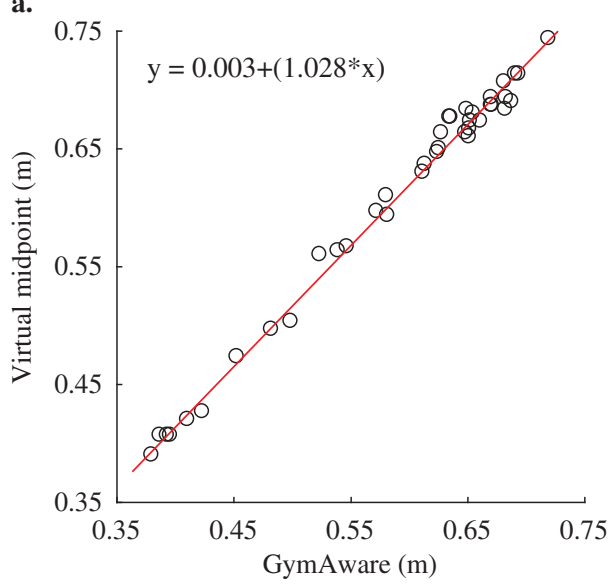

c.

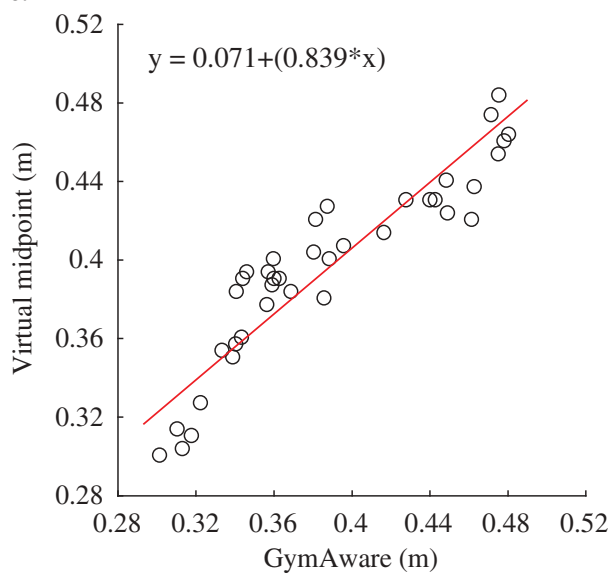

e.

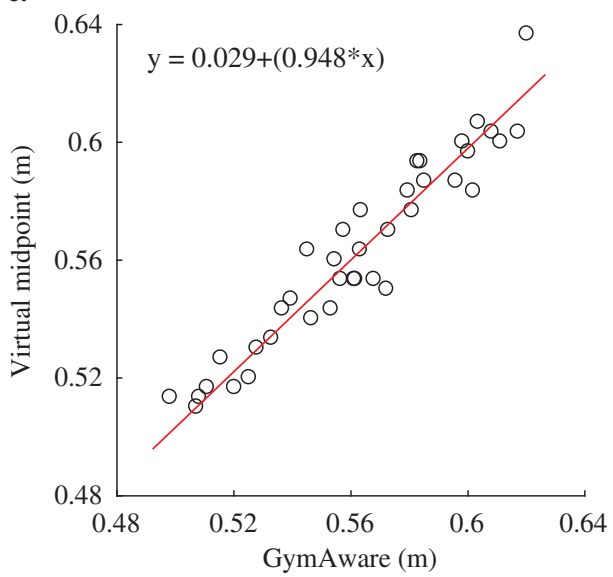

b.

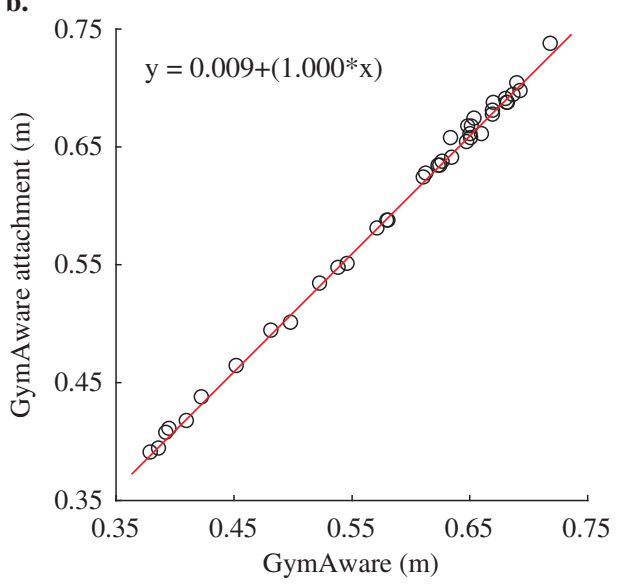

d.
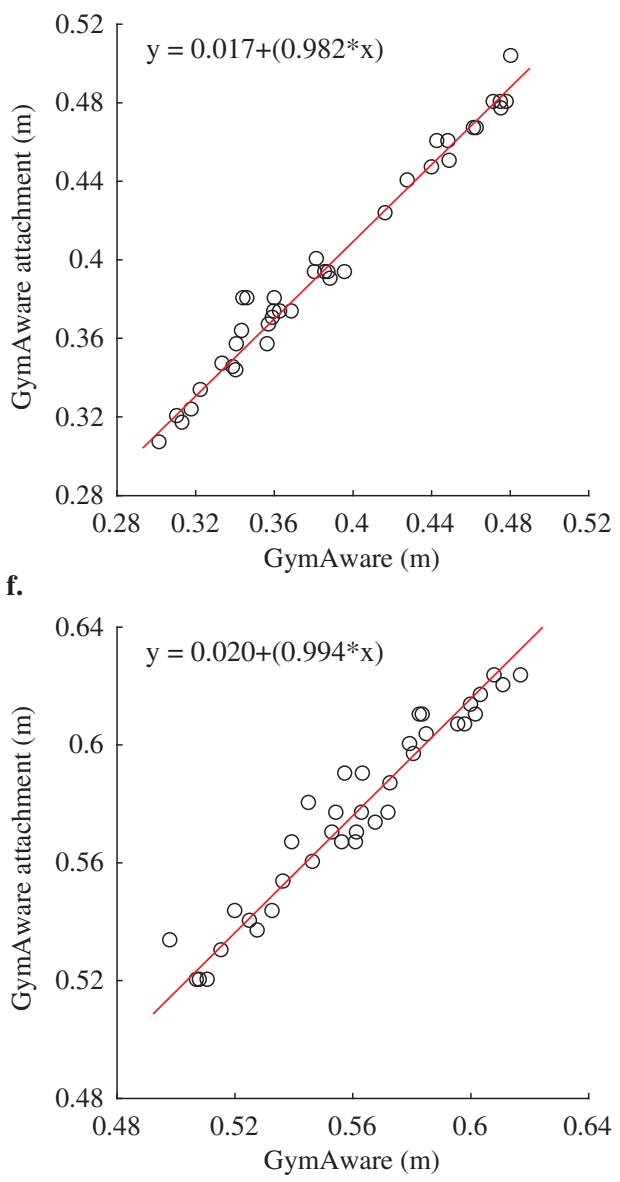

Figure 2. Least products regression displacement comparisons from GymAware to virtual midpoint $(a, c, e)$ and GymAware attachment (b, d, f). Back squat (a, b), bench press $(c, d)$ and deadlift $(e, f)$. 


\section{Reliability}

No significant differences were recorded for any of the variables between visits for the back squat or CMJ. In contrast, significant differences were observed for bench press between visits 3-2 for displacement (mean $\pm \mathrm{SD}$ : visit 3: $0.395 \pm 0.055 \mathrm{~m}$; visit 2: $0.383 \pm 0.053 \mathrm{~m}$;), with no significant differences recorded between other variables. For deadlift, significant differences were observed between visits 3-2 for displacement (mean \pm SD: visit 3: $0.557 \pm 0.034 \mathrm{~m}$; visit 2: $0.568 \pm 0.034 \mathrm{~m}$ ), with no significant differences found between other variables. The mean TE for all variables between visits was low to moderate (Table 2; range $0.6-8.8 \%$ ), with SWC ranging from 1.7-7.7\%; (back squat: 5.4-6.3\%; bench press: $4.4-5.5 \%$; deadlift: $1.7-7.7 \%$; CMJ: $6.0 \%$ ).

\section{Sample-size estimation}

For a simple test re-test or crossover design, minimum samplesizes were estimated as four (back squat and bench press), and 11 (deadlift) to enable detection of $80 \%$ power. If a control group is implemented into the research, sample-size estimations increase to 16 (back squat), 15 (bench press), and 44 (deadlift). For CMJ, a test re-test design would require a minimum of six participants, with inclusion of a control group increasing this to 25 .

\section{Discussion}

This was the first study to explore the validity and reliability of the GPT against integrated criterion devices. The results demonstrate that the GPT is a valid device for determining kinetic and kinematic variables during resistance training movements (back squat, bench press, deadlift, and CMJ) in a trained population. Least products regression between the criterion devices and the GPT resulted in high $R^{2}$ vales $(\geq 0.91)$ for 20 of the 23 comparisons. Furthermore, the GPT produced low to moderate TE $(0.6-8.8 \%)$ between visits, displaying high levels of intra-session reliability.

Within the data presented, the only measurements that showed a substantial difference between the GPT and criterion device were mean velocity for the deadlift. Moderate

Table 2. Typical error displayed as a percentage (TE) across variables between visits (V).

\begin{tabular}{llll}
\hline & TE V2-V1(\%) & TE V3-V2 (\%) & Mean TE (\%) \\
\hline Back squat & & & \\
$\quad$ Displacement & $3.9(2.9-5.9)$ & $3.7(2.7-5.6)$ & $3.8(3.0-5.3)$ \\
Peak velocity & $8.9(6.7-13.8)$ & $7.1(5.4-11.0)$ & $8.1(6.4-11.5)$ \\
Mean velocity & $7.9(5.9-12.3)$ & $6.0(4.5-9.3)$ & $7.0(5.6-10.0)$ \\
$\quad$ Peak force & $5.2(3.9-8.1)$ & $3.1(2.3-4.7)$ & $4.3(3.4-6.1)$ \\
$\quad$ Mean force & $0.6(0.4-0.9)$ & $0.7(0.5-1.0)$ & $0.6(0.5-0.9)$ \\
Bench press & & & \\
$\quad$ Displacement & $3.4(2.6-5.2)$ & $2.4(1.8-3.7)$ & $3.0(2.3-4.1)$ \\
$\quad$ Peak velocity & $5.6(4.2-8.6)$ & $6.8(5.1-10.4)$ & $6.2(4.9-8.7)$ \\
Mean velocity & $7.1(5.3-11.0)$ & $7.7(5.7-11.9)$ & $7.4(5.8-10.5)$ \\
Deadlift & & & \\
$\quad$ Displacement & $1.9(1.4-2.9)$ & $2.0(1.5-3.1)$ & $2.0(1.6-2.7)$ \\
$\quad$ Peak velocity & $10.1(7.5-15.6)$ & $7.4(5.5-11.4)$ & $8.8(7.0-12.5)$ \\
$\quad$ Mean velocity & $7.3(5.4-11.2)$ & $6.6(5.0-10.2)$ & $7.0(5.5-9.8)$ \\
$\quad$ Peak force & $2.4(1.8-3.6)$ & $3.7(2.8-5.7)$ & $3.1(2.5-4.4)$ \\
$\quad$ Mean force & $1.5(1.1-2.3)$ & $1.6(1.2-2.5)$ & $1.6(1.3-2.2)$ \\
CMJ & & & \\
$\quad$ Height & $6.7(5.0-10.4)$ & $3.6(2.7-5.6)$ & $5.4(4.3-7.7)$ \\
\hline
\end{tabular}

correlations were present for both $G A\left(R^{2}=0.54\right)$ and VM $\left(R^{2}=0.69\right)$ when compared directly to the GPT values. One practical explanation for this pertains to the sensitivity of the motion capture system (i.e. higher sampling frequency), as it was noted that with the deadlift an earlier concentric start point was often detected within the motion capture data. During the initial start phase of the deadlift, the vertical movement of the bar is minimal, as supported by the strong correlations present between the GPT and motion capture system for deadlift displacement $\left(R^{2} \geq 0.92\right)$. However, the addition of data points before the "true start" of the lift in the calculated velocity data, will result in a lower mean. The mean takes the sum of all data points and divides this by the number of points. Therefore, the inclusion of extra data points, which have a low velocity (minimal change in the rate of displacement), results in a lower mean. This is apparent from the lower mean velocities reported between the GPT and comparison values obtained from motion capture data (mean $\pm \mathrm{SD}$ : GPT: $0.432 \pm 0.041 \mathrm{~m} \cdot \mathrm{s}^{-1} ;$ GA: $0.332 \pm 0.054 \mathrm{~m} \cdot \mathrm{s}^{-1}$; VM: $0.401 \pm 0.048 \mathrm{~m} \cdot \mathrm{s}^{-1}$ ). One explanation may be associated with individual differences within the lift set-up. Tension is often placed upon the bar prior to initiation of the deadlift, causing the bar to flex or rotate (Hales, 2010). This would cause the GA (centred for the deadlift) and/or the VM to appear to displace vertically, triggering the data analysis process to register premature movement, prior to the GPT tether unwinding. This implies that the reported errors are due to the process of identifying the beginning of the movement, rather than the validity of the GPT. No current literature is available on the validity of an LPT when measuring mean velocity of a deadlift, meaning there is little evidence to provide support for this theory. Further research may wish to explore the role of the different methods of lift start identification on outcome variables, particularly with applied devices used to measure performance in the field.

With the exception of the mean velocity of the deadlift, the validity assessments have all shown strong correlations between the GPT and criterion measures for kinematic variables (Table 1). This appears to agree with similar research which has explored the validity of kinematic measures obtained via other commercially available LPTs. One such study investigated the validity of the Tendo Weightlifting Analyser during free-weight back squat and bench press (Garnacho-Castaño, López-Lastra, \& Maté-Muñoz, 2015). Results obtained from the LPT were validated against an isoinertial dynamometer, with high correlations for both peak and mean velocity reported, $R^{2}=0.92$ and 0.96 , respectively. These strong correlations show comparable results and provide further evidence for LPTs as a suitable monitoring tool for kinematic performance variables.

The kinetic variables calculated from the GPT are all derived from the collected position data. Differentiation causes errors in a signal to be magnified, therefore care should be taken to minimise noise in original data and ensure suitable smoothing methods are used. The GAP employs a down sampling method to minimise these errors. Strong correlations were found for peak and mean force of the squat and deadlift trials $\left(R^{2} \geq 0.94\right)$ suggesting that minimal errors were present in the post-processed GAP data. Crewther et al. (2011) explored the 
validity of kinetic variables during weighted squat jumps collected using the GPT and force plate. Moderate correlations were reported $\left(R^{2}=0.59-0.87\right)$ following comparisons between collection methods. Rather than increased error due to data processing, it was suggested that the lower values reported $\left(R^{2}=0.59\right.$; obtained during $20 \mathrm{~kg}$ jumps), were due to horizontal bar movement, which was reduced as resistance was increased (40 kg: $R^{2}=0.83 ; 60 \mathrm{~kg}$ and $80 \mathrm{~kg}: \mathrm{R}^{2}=0.87$ ). During this study, the GPT was attached to the end of the bar, increasing the potential for horizontal sway during jumping actions at lower resistances. While a similar attachment site was used in the present study, the inclusion of greater resistance, and therefore the removal of the ballistic nature of the movement likely decreased the potential for horizontal sway, increasing correlations between measurement devices (peak force: $R^{2}=0.97-0.99$; mean force: $R^{2}=0.94-0.99$ ). It is worth considering that the data collected via the GPT is that of the movement of the tether, meaning results can differ depending on lift technique (e.g. horizontal bar movement) and tether attachment site. It is therefore recommended that care be taken when selecting attachment sites, particularly at lower resistances, or when ballistic movements are employed, as this may lead to a greater presence of error, and thus skewing of data.

The second aim of this study was to establish the test-retest reliability of the GPT. For back squat and CMJ, the lack of significant differences and low to moderate TE suggest that the GPT is a reliable tool for collecting performance variables of resistance trained individuals performing these tasks. Similar results were reported for deadlift, with the exception of displacement, where significant differences were found between visits three and two. As all subsequent variables are derived from displacement, the presence of statistical difference between visits highlights concerns with the GPTs ability to measure this particular movement. However, the presence of low TE (2.0\%) between displacement data, and the absence of statistical difference reported between other visits and derived measures, suggest the GPT is a reliable tool for the measurement of deadlift variables. To the author's knowledge, no other research is available to provide evidence as to whether the results for these movements are comparable across other LPTs or participant groups. As such, it is suggested that future research further explores the test re-test reliability associated with the GPT.

Analysis of the bench press resulted in the presence of statistical difference between visits 3-2 for displacement only. While these results raise doubt regarding intra-visit reliability of the GPT, the minimal TE between visits (3.0\%), and the presence of no further statistical difference between derived measures alleviate these concerns. While currently no research exists regarding the reliability of displacement when utilising LPT technology, a similar study explored the reliability of peak velocity obtained via a comparable device during repeated bench press trials (Tendo Weightlifting Analyser; (Stock, Beck, DeFreitas, \& Dillon, 2011)). Peak velocity was recorded between 10-90\% 1RM, over two repeated visits. The results indicated that at lower resistances ( $\leq 70 \% 1 \mathrm{RM})$, test-retest reliability was moderate to high ( $\mathrm{CV}=3.1-5.8 \%)$, however, as resistance increased (> 70\% 1RM), relative consistency decreased ( $C V=10.3-12.6 \%)$. The authors suggested that this reduced consistency was likely associated with the low movement velocity present during the higher resistance trials, the devices ability to detect small differences in displacement, and potential participant fatigue due to previous repetitions. Within the current research, the results presented produced lower values than those reported $(\mathrm{CV}=6.2 \%)$ considering the resistance utilised $(80 \% 1 \mathrm{RM})$. This is potentially due to the precision of the GPTs displacement detection in relation to the Tendo Weightlifting Analyser $(0.3 \mathrm{~mm}$ versus $10.0 \mathrm{~mm}$ respectively), and the minimal stress placed on the participants prior to their repetitions. These results provide evidence supporting the use of the GPT within the monitoring of kinetic variables.

\section{Conclusion}

The aims of this research were to investigate and establish the validity and reliability of the GPT. The results presented show that the GPT provides valid measures of displacement and subsequent derivatives across a range of common resistance training exercises, when performed by trained individuals. Furthermore, low to moderate TE outputs, following repeated visits, provide confidence that the GPT can be utilised to detect worthwhile changes in performance within a trained participant group. The results do suggest care should be taken when monitoring deadlift performance, with peak velocity potentially offering a more robust measure than mean velocity. Future research may wish to investigate the source of the errors associated with the calculation of deadlift mean velocity, and the effect different methods of lift start identification have on this variable. Furthermore, as sample-size for future research has been estimated, researchers can use this information within the design subsequent research.

\section{Disclosure statement}

No potential conflict of interest was reported by the authors.

\section{ORCID}

Harry F. Dorrell (D) http://orcid.org/0000-0001-5394-971X Joseph M. Moore (D) http://orcid.org/0000-0001-5682-6897

\section{References}

Argus, C., Gill, N., \& Keogh, J. (2012). Characterization of the differences in strength and power between different levels of competition in rugby union athletes. The Journal of Strength \& Conditioning Research, 26(10), 2698-2704.

Argus, C., Gill, N., Keogh, J., \& Hopkins, W. (2011). Acute effects of verbal feedback on upper-body performance in elite athletes. The Journal of Strength \& Conditioning Research, 25(12), 3282-3287.

Argus, C., Gill, N., Keogh, J., Hopkins, W., \& Beaven, C. (2010). Effects of a short-term pre-season training programme on the body composition and anaerobic performance of professional rugby union players. Journal of Sports Sciences, 28(6), 679-686.

Baechle, T. R., \& Earle, R. W. (2000). Essentials of strength training and conditioning. Champaign, IL: Human Kinetics.

Beaven, C., Cook, C., Kilduff, L., Drawer, S., \& Gill, N. (2012). Intermittent lower-limb occlusion enhances recovery after strenuous exercise. Applied Physiology, Nutrition, and Metabolism, 37(6), 1132-1139. 
Conceição, F., Fernandes, J., Lewis, M., Gonzaléz-Badillo, J., \& Jimenéz-Reyes, P. (2016). Movement velocity as a measure of exercise intensity in three lower limb exercises. Journal of Sports Sciences, 34(12), 1099-1106.

Cormie, P., Mccaulley, G., Triplett, N., \& Mcbride, J. (2007). Optimal loading for maximal power output during lower-body resistance exercises. Medicine \& Science in Sports \& Exercise, 39(2), 340-349.

Crewther, B., Kilduff, L., Cunningham, D., Cook, C., Owen, N., \& Yang, G. (2011). Validating two systems for estimating force and power. International Journal of Sports Medicine, 32(4), 254-258.

Crewther, B., Kilduff, L., Cunningham, D., Cook, C., \& Yang, G. (2010). Validity of two kinematic systems for calculating force and power during squat jumps. British Journal of Sports Medicine, 44(14), i26.

Cronin, J., Hing, R., \& Mcnair, P. (2004). Reliability and validity of a linear position transducer for measuring jump performance. The Journal of Strength \& Conditioning Research, 18(3), 590-593.

Drinkwater, E., Galna, B., McKenna, M., Hunt, P., \& Pyne, D. (2007) Validation of an optical encoder during free weight resistance movements and analysis of bench press sticking point power during fatigue. The Journal of Strength \& Conditioning Research, 21(2), 510-517.

Drinkwater, E., Lawton, T., Lindsell, R., Pyne, D., Hunt, P., \& McKenna, M. (2005). Training leading to repetition failure enhances bench press strength gains in elite junior athletes. The Journal of Strength \& Conditioning Research, 19(2), 382-388.

Drinkwater, E., Moore, N., \& Bird, S. (2012). Effects of changing from full range of motion to partial range of motion on squat kinetics. The Journal of Strength \& Conditioning Research, 26(4), 890-896.

Garnacho-Castaño, M., López-Lastra, S., \& Maté-Muñoz, J. (2015). Reliability and validity assessment of a linear position transducer. Journal of Sports Science \& Medicine, 14(1), 128-136.

González-Badillo, J., Pareja-Blanco, F., Rodríguez-Rosell, D., Abad-Herencia, J., del Ojo-López, J., \& Sánchez-Medina, L. (2015). Effects of velocitybased resistance training on young soccer players of different ages. The Journal of Strength \& Conditioning Research, 29(5), 1329-1338.

González-Badillo, J., \& Sánchez-Medina, L. (2010). Movement velocity as a measure of loading intensity in resistance training. International Journal of Sports Medicine, 31(5), 347-352.

Haff, G. (2012). Training principles for power. Strength \& Conditioning Journal, 34(1), 2-12.

Hales, M. (2010). Improving the deadlift: Understanding biomechanical constraints and physiological adaptations to resistance exercise. Strength \& Conditioning Journal, 32(4), 44-51.
Hamill, J., Knutzen, K. M., \& Derrick, T. R. (2015). Biomechanical basis of human movement. Philadephia, PA: Wolters Kluwer.

Harries, S., Lubans, D., \& Callister, R. (2012). Resistance training to improve power and sports performance in adolescent athletes: A systematic review and meta-analysis. Journal of Science and Medicine in Sport, 15(6), 532-540.

Harries, S., Lubans, D., \& Callister, R. (2015). Systematic review and metaanalysis of linear and undulating periodized resistance training programs on muscular strength. The Journal of Strength \& Conditioning Research, 29(4), 1113-1125.

Hopkins, W. (2000). Measures of reliability in sports medicine and science. Sports Medicine, 30(1), 1-15.

Hopkins, W. (2015). Spreadsheets for analysis of validity and reliability. Sportscience, 19(1), 36-42.

Jidovtseff, B., Harris, N., Crielaard, J., \& Cronin, J. (2011). Using the loadvelocity relationship for 1 RM prediction. The Journal of Strength \& Conditioning Research, 25(1), 267-270.

Kraemer, W., \& Ratamess, N. (2004). Fundamentals of resistance training: Progression and exercise prescription. Medicine and Science in Sports and Exercise, 36(4), 674-688.

Lamas, L., Ugrinowitsch, C., Rodacki, A., Pereira, G., Mattos, E., Kohn, A., \& Tricoli, V. (2012). Effects of strength and power training on neuromuscular adaptations and jumping movement pattern and performance. The Journal of Strength \& Conditioning Research, 26(12), 3335-3344.

Pereira, M., \& Gomes, P. (2003). Movement velocity in resistance training. Sports Medicine, 33(6), 427-438.

Peterson, M., Rhea, M., \& Alvar, B. (2004). Maximizing strength development in athletes: A meta-analysis to determine the dose-response relationship. The Journal of Strength \& Conditioning Research, 18(2), 377-382.

Sanchez-Medina, L., \& González-Badillo, J. (2011). Velocity loss as an indicator of neuromuscular fatigue during resistance training. Medicine \& Science in Sports \& Exercise, 43(9), 1725-1734.

Sánchez-Medina, L., Pallarés, J., Pérez, C., Morán-Navarro, R., \& González-Badillo, J. (2017). Estimation of relative load from bar velocity in the full back squat exercise. Sports Medicine International Open, 1(2), 80-88.

Stock, M. S., Beck, T. W., DeFreitas, J. M., \& Dillon, M. A. (2011). Test-Retest reliability of barbell velocity during the free-weight bench-press exercise. The Journal of Strength \& Conditioning Research, 25(1), 171-177. 